\title{
A summer fellowship with Albert Ellis
}

\author{
Anthony J. Mander
}

Albert Ellis formulated what is now known as rational emotive behaviour therapy (REBT) in 1955. The Institute for Rational Emotive Therapy (IRET), a not-for-profit organisation chartered by the Regents of the University of the State of New York, opened in its current mid-town Manhattan site in 1965.

Despite the cognitive-behavioural roots of REBT and the publication of Ellis' work a decade or so earlier than Beck, his theories have not received such widespread acceptance in psychiatry in the United Kingdom. There are a number of reasons for this which include the lack of REBT specific research (which has been recognised and addressed by the IRET over the last six years) and Ellis' style, which at least in the setting of a demonstration can come over as abrasive and confrontational. This relative neglect of his contribution is apparent in the otherwise excellent review of important works in the field of cognitive behaviour therapy by Scott (1994) which fails to mention Ellis or any of the other prominent contributors to REBT. To some extent this was remedied in the same Journal with a review of Reason and Emotion in Psychotherapy (Ellis, 1962) by Dryden (1994), a text which is currently being revised.

In 1992 the IRET started to award six summer fellowships to allow college faculty members to spend three weeks at the Institute to review their skills and knowledge in the theory and practice of REBT. A major aim of these fellowships is to produce individuals who will in turn teach high quality REBT to their own trainees and to stimulate a wider research base. I was fortunate enough to be awarded one of these fellowships between 4-27 July 1994.

\section{What is REBT?}

Ellis originally referred to his therapeutic process as rational therapy, later to be called rational emotive therapy to ensure that the importance of emotions was not overlooked.
The change of name to rational emotive behaviour therapy in 1993 acknowledged the significant role that behavioural interventions have always played in the REBT therapist's armamentarium. It is one of the 17 or so different therapies that rightly belong under the generic term cognitive therapy and was the forerunner of the constructivist model. The different cognitive therapy schools have much in common although REBT has made some unique contributions. These include the centrality of demandingness in emotional disturbance ('the tyranny of the shoulds', 'musturbation', 'hardening of the oughteries'), unconditional self acceptance (as opposed to supporting self esteem through positive self rating) and a philosophy of life that goes beyond the treatment of psychological problems and is best seen as long range hedonism.

REBT concentrates on what Ellis terms the 'elegant solution' aimed at the individual's underlying philosophy (Appendix). It is useful to consider that between awareness and appraisal of an event, there are a series of cognitive layers of increasing depth.

(a) Self statements, i.e. internal dialogue of which the individual is aware.

(b) Cognitive styles, i.e. processing elements such as arbitrary influence, selective abstraction etc.

(c) Schema, i.e. assumptive worlds, cognitive maps, life theories, tacit knowledge etc.

Beck's interventions would therefore focus on the self statements and styles (which REBT terms 'inelegant'). Ellis has the deeper schema as the focus of treatment on the basis that individuals will be less emotionally disturbed on the occasions when (as in the example in the Figure) their initial assumption is correct so long as they do not hold the core schema that it is necessary to be liked and awful if 

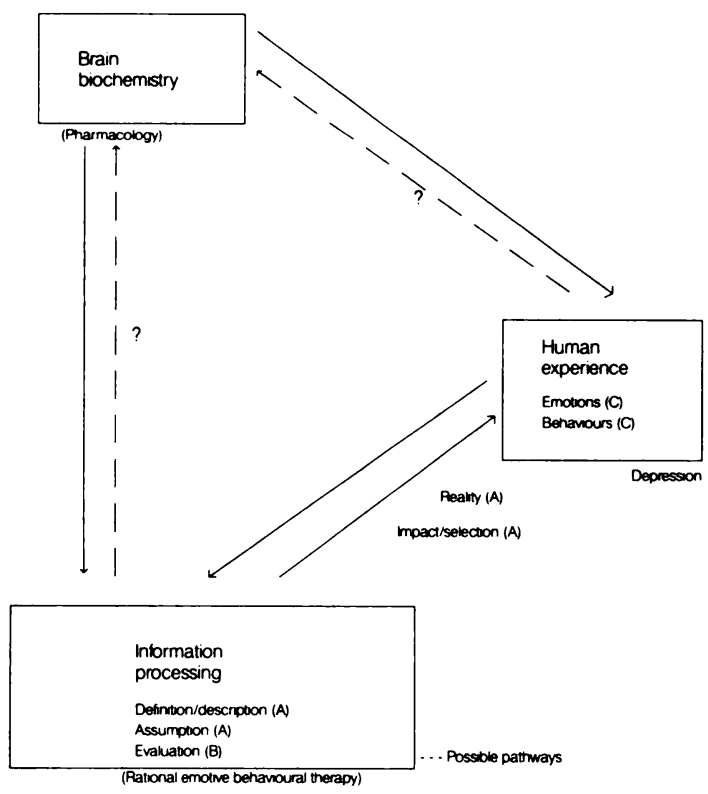

Fig. 1. Putative constructivist model linking psychological and biochemical factors

people are angry. In practice, there is substantial overlap in the way therapists of each school conduct treatment.

Of particular attraction to psychiatry is the explicit acknowledgement by Ellis of the prime role played by human biology. Hence, experience is not sufficient on its own to cause disturbance. It is the interaction of this with the person's biologically orientated demands. As Dryden (1994) has stated, REBT clearly regards biology as the major factor in producing emotional disturbance but Ellis gives people primary control of their emotional reins in perpetuating this disturbance. REBT actively encourages the combined use of psychological and pharmacological treatment where appropriate.

A hypothetical model of the interaction between biological and psychological variables and their influence on human experience is outlined in Fig 1 . In any given individual the relative contribution of these factors will vary. It follows that all therapeutic strategies attempt to change biology, information processing, or both.

\section{The Institute}

This provides low cost clinical services using individual and group formats. Corporate services advise industry and there is a special interest in biofeedback. A wealth of books and tapes are available for patients and trainee therapists. Part-time training is offered to interns and fellows over a two year period requiring approximately 20 hours per week which includes more than four hours of supervision from a staff therapist. External training can be obtained through the Primary, Advanced and Associate Fellowship Programmes (details from IRET, 45 East 65th Street, New York, NY 10021-6593; telephone 212-535-0822, fax 212-249-3582).

Ellis still works a punishing schedule, lending credence to his claim that he has seen more clients than any other therapist in history. In addition to teaching, writing and seeing individual clients, he runs two groups each evening Monday to Wednesday between 6.00 to 9.00 p.m. and still performs a public demonstration on Fridays between 8.00 and 10.00 p.m.

\section{The Summer Fellowship}

This consists of lectures, seminars, individual therapy, case supervision and attendance on a regular basis as a co-therapist in one of Ellis' groups.

In general, the programme consists of a two and $a$ half hour session each morning and afternoon with several evening sessions scheduled for seminars, demonstrations or attendance at groups. There are only two free days during the programme. The Fellowship enables completion of the Primary and Advanced practicums each running over four days. Lecture and seminar topics include homosexual issues, addictions, biofeedback, sexual assault, anger, bereavement, sex roles, personality disorders, career counselling and research issues. However, the highlight is undoubtedly the opportunity to spend a significant amount of time with Ellis which includes him discussing his life, family, career and development of REBT. While some of this is available in the literature, his willingness to self disclose enables a much greater understanding of the therapy process. He also delivers powerful insights into the lives and works of other pioneers in the fields of psychiatry and psychology from the 1930s onwards, most of whom were personally known to him. Training in the practical aspects of delivering REBT has changed over the last three years. Small groups of six to eight participants act alternately as patient and therapist. 
Role play is strongly discouraged on the basis that all people have emotional problems that can be dealt with in these sessions and provide a more realistic experience for the trainee therapist. The group is supervised by a staff therapist and is the most popular part of the training experience. This format has replaced the previous method which utilised the use of tapes made by the participants. The 'live' method is more flexible and effective, allowing immediate intervention by the supervisor if necessary. From the Associate Fellowship onwards supervision is of tapes of actual therapy sessions with patients.

\section{Benefits}

There is a real need in psychiatry to produce professionals with a thorough and complete training in cognitive behaviour therapy. Because the theories of Ellis and others seem superficially straightforward and books have been published on the practical aspects of treatment delivery (e.g. Hawton et al, 1989), a 'cookbook' mentality of treatment approaches is in danger of being developed. The New York Institute has always concentrated on the training of professionals as a major priority and there is now a world wide network of associated organisations.
Cognitive behaviour therapy is becoming a major treatment force and with its accent on an empirical approach, efficiency and rigorous assessment of outcome, it is likely to appeal to those who have to pay for services whether they be private sector insurers or NHS authorities. There is a need to develop recognised specialist qualifications within the college and for employers to consider the appointment of consultant psychotherapists with a detailed training in CBT. Much still needs to be done to integrate the clinical work of Beck and Ellis with experimental cognitive psychology if this field is to continue to develop further in a meaningful way.

\section{References}

DRYDEN, W. (1994) Books Reconsidered-Reason and Emotion in Psychotherapy: Albert Ellis. British Journal of Psychiatry, 164, 131-135.

Hawton, K., Salkovskis, P., KIRK J. et al (1989) Cognitive Behaviour Therapy for Psychiatric Problems. Oxford: Oxford University Press.

ScoTr. J. (1994) Reading About ... Cognittve Therapy. British Journal of Psychiatry. 164, 126-130.

Anthony J Mander, Clinical Senior Lecturer, University of Western Australia, Royal Perth Hospital, GPO Box X2213, Western Australia 6001 and Director, Institute for Cognitive Behaviour Therapy, GPO Bax A41, Western Australia 6000

\section{Appendix}

Information processing

(a) Activating event

(i) Reality
(ii) Impact/selection
(iii) Definition/description
(iv) Assumption

(b) Beliefs (rational or irrational)

Evaluation

(c) Consequences

Affect

Behaviour
Friend does not speak

Observer notices

Awareness and thought about event.

"My friend is angry with me."*

*Automatic thoughts targeted in Beck's CBT approach.

"**Irrational beliefs targeted in Ellis' REBT method. 